\title{
The quiet Sun magnetic field: statistical description from THEMIS observations ${ }^{\star}$
}

\author{
V. Bommier \\ LESIA, Observatoire de Paris, CNRS-INSU-UMR8109, UPMC Univ. Paris 06, Université Paris Diderot - Paris 7 , \\ 5 Place Jules Janssen, 92190 Meudon, France \\ e-mail: V.Bommier@obspm.fr
}

Received 15 September 2010 / Accepted 30 March 2011

\begin{abstract}
Context. The pioneer interpretation of Hanle-effect depolarization observed at the limb was the one of a turbulent nature for the solar internetwork photospheric magnetic field. In the present paper, we propose an alternative interpretation of these measurements, which are now complemented with Zeeman-effect observations to allow one to conclude on the properties of weak fields.

Aims. In a previous paper, we presented an internetwork field diagnostic from data obtained with the ZIMPOL II polarimeter mounted on the THEMIS telescope. In the present paper, we present the results obtained with the THEMIS polarimeter with ten times more pixels. The agreement between the results obtained with the two polarimeters confirm the goodness of both the THEMIS polarimeter measurements and our data treatment.

Methods. The Zeeman-effect measurements are interpreted via a 2-component model, which has a field-free component and a magnetized one, filling a fraction of the analyzed pixel equal to $1-\alpha$ and $\alpha$, respectively. We determine this $\alpha$ parameter independently of the magnetic inversion. We already applied these methods to the previous interpretation of the ZIMPOL/THEMIS data.

Results. The magnetic field strength and magnetic field inclination are not independent, because the strongest fields are more vertical and the weakest fields are more horizontal, both in ZIMPOL/THEMIS and THEMIS/THEMIS data.

Conclusions. This suggests that the photospheric internetwork field has the structure of scattered narrow fluxtubes consisting in a vertical field, which weakens in opening - widening with individual field line bending - with height. The weakest fields then have a 2D horizontal structure, instead of the usually admitted 3D turbulent one. This does not contradict the previous Hanle-effect observations, because it is insensitive to vertical fields. The number of fluxtubes inside each pixel (which was 0.21 arcsec for the THEMIS/THEMIS and 0.53 arcsec for the ZIMPOL/THEMIS data) should, however, remain small. We could make a conclusion about this after observing a non-zero spatial correlation of the magnetic field orientation, with an estimated correlation length of $250 \mathrm{~km}$.
\end{abstract}

Key words. magnetic fields - polarization - Sun: magnetic topology - Sun: photosphere - techniques: polarimetric - techniques: spectroscopic

\section{Introduction}

The nature of the magnetic field in internetwork regions was revealed in the pioneer work by Stenflo (1982), where the limb linear polarization of several lines was interpreted in terms of scattering polarization and magnetic depolarization (Hanle effect) by a turbulent field. The turbulent nature of the field was derived from the absence of rotation of the polarization direction (the second Hanle-effect feature). Due to the nonlinearity of the Hanle effect, a global depolarization remains in this unresolved mixed polarity scheme. Stenflo (1982) was able to expound upon the magnetic depolarization in the observations, to ascribe it to a turbulent field, and to model the Hanle depolarization by such a field. The density matrix version of this theoretical depolarization can be found in Trujillo Bueno \& Manso Sainz (1999, Appendix A). The first quantitative interpretation for Sr I $\lambda 4607 \AA$ can be found in Faurobert-Scholl (1993), later on improved for the competing collisional depolarization (FaurobertScholl et al. 1995). Improved measurements were analyzed by

* Based on observations made with the French-Italian telescope THEMIS operated by the CNRS and CNR on the island of Tenerife in the Spanish Observatorio del Teide of the Instituto de Astrofísica de Canarias.
Bommier et al. (2005), and a review of measurements and analysis results can be found in Trujillo Bueno et al. (2004).

More recently, the internetwork magnetic field has also become accessible by interpretating the Zeeman effect. But in contrast to the Hanle effect, the Zeeman effect is linear in magnetic field, so that such an accessibility results from improvement in spatial resolution, which now allows one to resolve the magnetic structuring on subarcsecond scales. Because it is linear, the Zeeman effect is sensitive to both weaker and stronger fields, unlike the Hanle effect that saturates in strong fields. Thus, by using the Zeeman effect, one has access to stronger fields possibly present in the internetwork, which remain invisible with the Hanle effect. Interesting results were obtained in the near-IR domain, where the Zeeman effect is more sensitive to the magnetic field strength (Khomenko et al. 2003; Martínez González et al. 2008). The studies using the visible Fe I $6301.5 \AA$ and $6302.5 \AA$ spectral lines, which show a stronger field in the $\mathrm{kG}$ range (Socas-Navarro \& Sánchez Almeida 2002; Domínguez Cerdeña et al. 2003; Socas-Navarro et al. 2004; Lites \& SocasNavarro 2004), have been challenged (Martínez González et al. 2006; López Ariste et al. 2007; Khomenko \& Collados 2007). The availability of HINODE data has led to a new analysis, that concludes that hG fields are the most frequent (Orozco Suárez et al. 2007). The analysis was reinforced by further investigation 
of the inversion validity (del Toro Iniesta et al. 2010), and confirmed by another inversion method (Asensio Ramos 2009). However, a different analysis of HINODE data has recently been proposed (Viticchié et al. 2011), with the MIcro-Structure Magnetic Atmopshere (MISMA) model, which proves the coexistence of $\mathrm{hG}$ and $\mathrm{kG}$ fields in the internetwork. Indeed, the field remains partly unresolved, and only a statistical description of the field can be attained by interpretating data (Martínez González et al. 2010b). The tool in this statistical description is the probability density function. Bommier et al. (2009) proposed a clarification of its definition, but it has to be kept in mind that the probability remains intimately linked to the model used for data interpretation.

In the same paper the authors presented the results of Zeeman-effect observations made with the ZIMPOL II polarimeter mounted on the THEMIS telescope (abbreviated as ZIMPOL/THEMIS in the following). These data were made of 1680 analyzed solar pixels (1680 spectra). The present paper reports the results obtained by applying the analysis method adopted in Bommier et al. (2009) for analyzing ZIMPOL/THEMIS data, to a ten times larger dataset observed earlier with the THEMIS analyzer instead (19470 pixels and/or spectra, denoted as THEMIS/THEMIS data). As the results are found to agree, this confirms the goodness of both the THEMIS polarimeter measurements and our data treatment.

The paper is organized as follows. The THEMIS/THEMIS observations are described in Sect. 2. The first step in the data treatment, the polarimetric analysis, is described in Sect. 3. We point out a recentering technique that we applied to our data to free them from velocity changing during the beam exchange process. The UNNOFIT inversion results are given in Sect. 4. However, as described in Bommier et al. (2007) and following the tests performed in that paper, the inversion is unable to discriminate between the magnetic field strength and the magnetic filling factor. Only their product, the local average magnetic field, is recovered by the inversion. The technique has to be complemented by another one to achieve the discrimination. We applied the same technique as proposed in Bommier et al. (2009) to determine the magnetic filling factor order of magnitude from spectropolarimetric data combination, nearly independently of the inversion. The results are presented in Sect. 5. Section 6 is devoted to determining a spatial correlation length of the magnetic field vector inclination, in the ZIMPOL/THEMIS and THEMIS/THEMIS data. Finally, a dependency relation is revealed between the field strength and the field inclination, which leads to renewing the internetwork magnetic-field reckoned model, as discussed in the concluding Sect. 7. In this Sect. 7, we come back to the discussion of why recent results lead to hG fields with a typical 20\% filling factor (Orozco Suárez et al. 2007; Asensio Ramos 2009), whereas we get kG fields having with a few percent filling factor, given by the maximum of the PDFs.

\section{Observations}

The observations were made on 25 July 2007. They consisted in small scans of ten steps of 0.3 arcsec, repeated 30 times in order to study the time evolution. The slit was oriented solar north and positioned at disk center. The cameras had been renewed, so that the pixel size was two times smaller than previously, namely 0.21 arcsec. The polarimeter was operating in grid mode, where the two polarization states after the beamsplitter $I+S$ and $I-S$ ( $S$ being one of the Stokes parameters $Q, U, V$ ) are alternated along the slit as spectral bands in the final output. The beam exchange was performed. The integration time was $120 \mathrm{~ms}$ and 10 accumulations were performed. Taking into account the camera readout and polarimeter actuation times, the total duration for a full Stokes polarimetry was of the order of $12 \mathrm{~s}$ per step. The polarimetric accuracy was $1.1 \times 10^{-3}$.

Four cameras were simultaneously used, repectively centered on $\mathrm{Na}_{\mathrm{I}} \mathrm{D}_{1}, \mathrm{Cr}$ I $\lambda 5781.8 \AA$ (a line that forms very low in the photosphere), Ca I $\lambda 6102.7 \AA$, and Fe I $\lambda 6301.5 \AA$ and $\lambda 6302.5 \AA$. Only this last line is analyzed in the following, as a first step. The results are presented for only one barrel of the grid, of 15 arcsec high, that was particularly quiet. This results in 19470 pixels and/or spectra analyzed. The TIP-TILT was ON.

\section{Polarimetric analysis}

The polarimetric analysis is the transformation of the telescope output into Stokes spectra. Our program APOLAR makes extensive use of the spectral line position method described in Bommier \& Rayrole (2002b). Its precision may be adjusted at the desired level, here $10^{-4}$ (accuracy on the line position), which was reached in about three iterations. The spectral shift technique by FFT phase shift is also used, including the apodization technique described in the same paper. With these tools, the flat field matrix is first computed, along with the spectra coalignment parameters (from flat field images). The demodulation technique is unnecessary for the polarization analysis. We assume that the polarimeter output consists in "pure" states $I \pm S$, where $S$ is one of the polarization Stokes parameters $Q, U, V$. Then, the polarization can be derived straight by the appropriate subtraction. We correct for the unavoidable channel differences by performing the beam exchange (Bommier \& Molodij 2002a), now available in all the Stokes parameters at THEMIS. The beam exchange introduces two different measurement times $t_{1}$ and $t_{2}$ for each Stokes parameter. Finally, we point out that the magnetic field does not shift the $I \pm Q$ and $I \pm U$ profiles spectrally. The eventual shift between these two measurements can then only result from solar or Earth atmosphere movements (even inside the spectrograph). To get rid of this effect, we realign the $I \pm Q$ and $I \pm U$ profiles before performing the beam exchange subtraction. As a result, the $Q$ and $U$ profiles approach their expected theoretical spectral symmetry in a reasonable manner. Final discrepancies may exist. Obviously, we do not apply this realignement to the $I \pm V$ profiles whose positions are sensitive to the magnetic field.

\section{Inversion results}

We applied the UNNOFIT inversion code (Bommier et al. 2007), which is based on applying the Marquardt algorithm to fit the observed Stokes profiles with theoretical ones stemming from the Unno-Rachkovsky solution of the polarized radiation transfer equation in the presence of a magnetic field. The distinctive feature of UNNOFIT is that the partial derivatives of the output signal as a function of the searched-for parameters, which are required by the Marquardt algorithm, are analytical. A magnetic filling factor has been introduced in the model, which is 2-component, one magnetic and one non-magnetic having all their other parameters identical. Nine parameters are derived: 1/ the magnetic field strength, 2/ the magnetic field inclination, 3/ the magnetic field azimuth (within $180^{\circ}$, the ambiguity is not resolved), 4/ the magnetic filling factor, 5/ the $a$ damping parameter of the Voigt function, 6/ the absorption profile Doppler width, $7 /$ the line absorption relative coefficient $\eta_{0}, 8 /$ the line spectral position (the radial velocity), and $9 /$ the $\beta$ parameter 

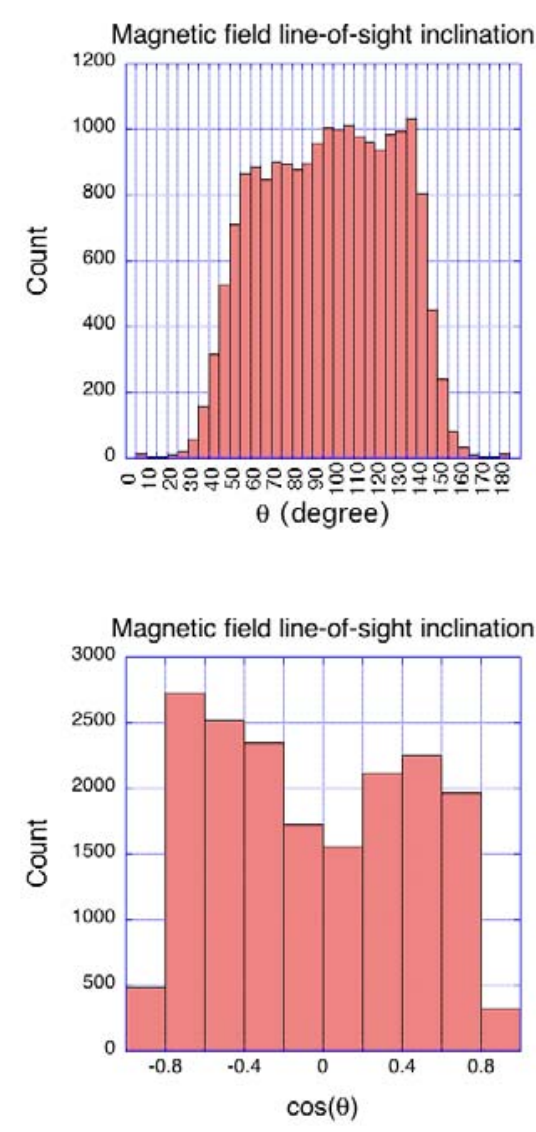

Fig. 1. Histogram of the inclination angle (top), and of the cosine of the inclination angle (bottom).

that describes the atmosphere following the Milne-Eddington approximation

$B_{\mathrm{P}}=B_{0}+B_{1} \tau_{\mathrm{c}}=B_{0}\left(1+\beta \tau_{\mathrm{c}}\right)$,

where $B_{\mathrm{P}}$ is the Planck function and $\tau_{\mathrm{c}}$ the continuum optical depth measured along the vertical. The two usual MilneEddington parameters $B_{0}$ and $B_{1}$ of this equation are different from the two field strengths $B_{0}$ and $B_{1}$ that we introduce in our Eqs. $(2,3)$ below. The inversion is repeated 20 times with random initialization, and the final best fit is kept. The code is OpenMP parallelized on the map pixels. The execution time is $35 \mathrm{mn}$ with eight processors (quadri-Opteron bi-cores or biXeon quad-cores). For the moment, we have not succeeded in setting a totally efficient single initialization, which would reduce the execution time considerably.

The histogram of the inclination angle (Fig. 1) has to be compared to Fig. 4 of Bommier et al. (2009). There is a difference in the central part of the histogram, where a central hollow exists in the ZIMPOL/THEMIS data histogram (Fig. 4 of Bommier et al. 2009), which does not exist in the THEMIS/THEMIS data histogram of Fig. 1. We ascribe this hollow to the non-zero circular polarization $V / I$ all along the slit in the ZIMPOL/THEMIS data, which is not the case for the THEMIS/THEMIS data. The final explanation of this behavior is not easy to find. Due to the systematic presence in the ZIMPOL/THEMIS data, we suspect there is a misalignment in the data reduction process, because misalignment results in $V$-shaped profiles. But we cannot say that for certain, because we did not examine the

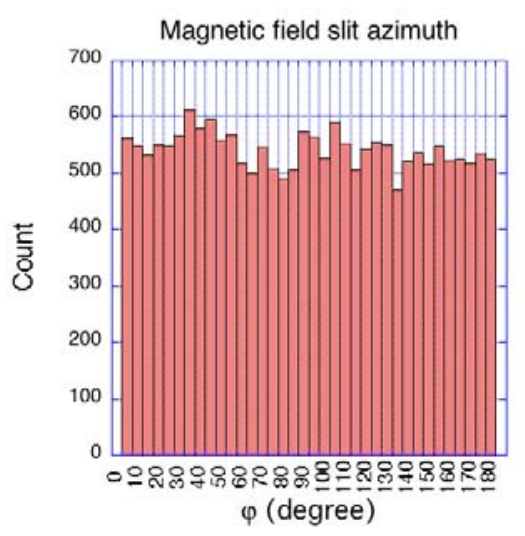

Fig. 2. Histogram of the azimuth angle (ambiguity unresolved).

ZIMPOL/THEMIS data reduction package in detail. Apart from this difference in the central hollow, both histograms display the sinusoidal shape of an isotropical distribution (related to the elementary volume element in spherical coordinates $\sin \theta \mathrm{d} \theta \mathrm{d} \varphi$ ). To confirm this shape, we plotted the histogram of the cosine of the inclination angle in the bottom of Fig. 1. It can be seen that all the inclinations are nearly equally probable, except for the very vertical ones. A possible explanation for the vertical fields not being retrieved by the inversion code probably is its attempt to interpret the noise level of the data in the linear polarization measurements. This will be interpreted as a weak polarization signal, i.e., an inclination different from $0^{\circ}$ (or $180^{\circ}$ ). The histogram of the azimuth angle (Fig. 2) is the one for an isotropical azimuth distribution, as observed with ZIMPOL (Fig. 5 of Bommier et al. 2009).

As described in Bommier et al. (2007), the magnetic field strength and filling factor are not separately recovered, only their product, the local average magnetic field strength, is recovered. The histogram of the longitudinal field (local average) is displayed in Fig. 3 and has to be compared to Figs. 7 and 16 of Bommier et al. (2009). The histogram shape is equally Gaussian, but the halfwidth is larger in the THEMIS/THEMIS data $(27 \mathrm{G})$ than in the ZIMPOL/THEMIS ones (13.5 G). This probably has to be related to the 0.21 arcsec pixel size in the THEMIS/THEMIS data, whereas it is 0.53 arcsec in the ZIMPOL/THEMIS data. The bottom part of Fig. 3 displays the same histogram on a logarithmic count scale, to make the wings more visible. No departure from the Gaussian shape in the wings appears in the THEMIS/THEMIS data, contrarily to what has been observed by other authors (see for instance Stenflo \& Holzreuter 2003) and to what is observed also by the ZIMPOL/THEMIS data (Fig. 16 of Bommier et al. 2009), but with a very small count number. We ascribe this absence of wings to the particularly quiet character of the region we observed, because we associate the presence of such wings to stronger network fields present in the field of view, which was not the case in our present observation.

The center of gravity (COG) method of Rees \& Semel (1979) could have been applied to the local average longitudinal field determination for a comparison with the UNNOFIT inversion results presented here. The unpublished result of the UNNOFIT test of Bommier et al. (2007) for the longitudinal field component gives excellent results, and the COG method is known to correctly apply to non-weak fields also, so that a very good 

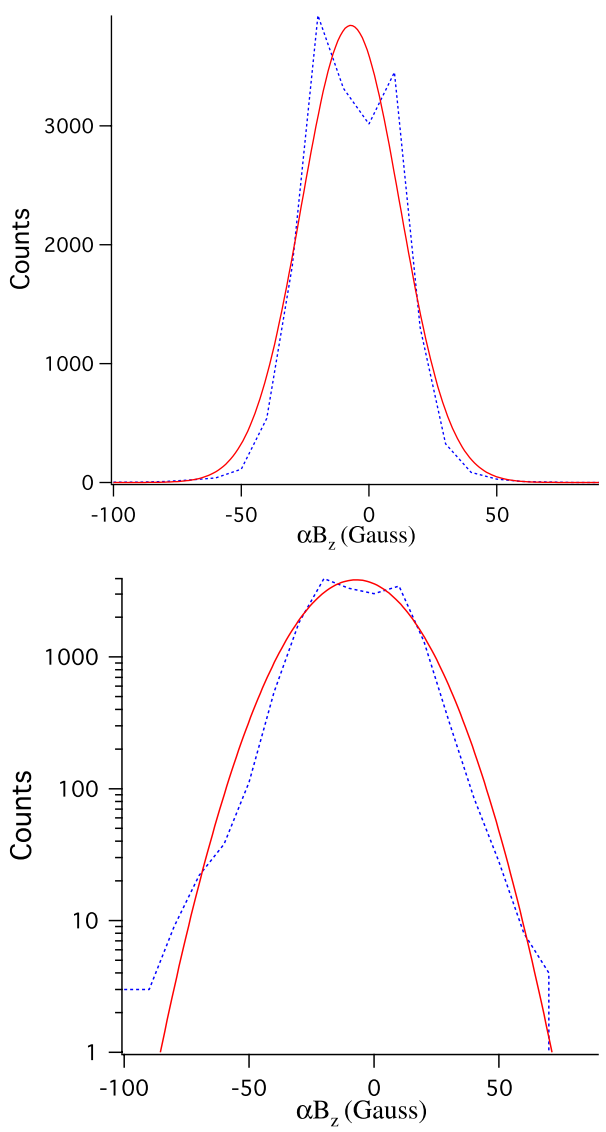

Fig. 3. Histogram of the local average longitudinal magnetic field histogram (dotted line) and fit by a Gaussian $\exp \left(-\left(\left(x-x_{0}\right) / w\right)^{2}\right)$ of halfwidth $w=27 \mathrm{G}$ (full line). Top: linear $Y$-scale (counts). Bottom: logarithmic $Y$-scale, to make the wings more visible.

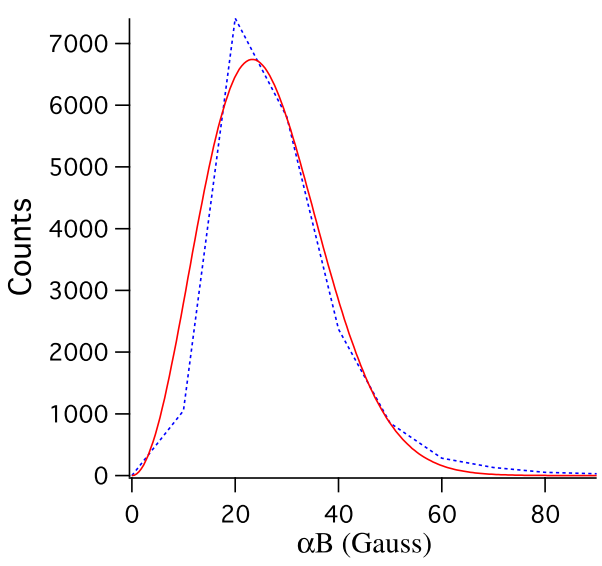

Fig. 4. Histogram of the local average magnetic field strength (dotted line) and fit by a Maxwellian $4 \pi x^{2} \exp \left(-(x / w)^{2}\right)$ of halfwidth $w=23 \mathrm{G}$ (full line).

agreement is found, and a detailed comparison of both methods will be the object of a later paper.

The histogram of the local average magnetic field strength, given in Fig. 4, is very similar to the one of the ZIMPOL/THEMIS data (see Fig. 8 of Bommier et al. 2009). Again, the only difference is the larger width in the case of the THEMIS/THEMIS data $(23 \mathrm{G})$ than in the ZIMPOL/THEMIS ones $(11 \mathrm{G})$. Again, we ascribe this behavior to the different pixel sizes of the two samples.

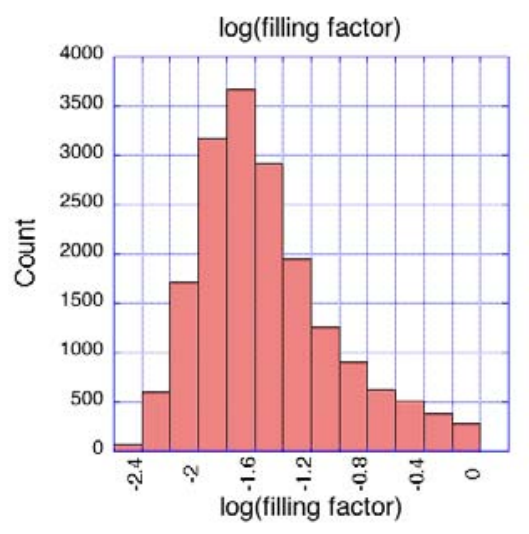

Fig. 5. Histogram of the magnetic filling factor $\alpha$ (in logarithm).

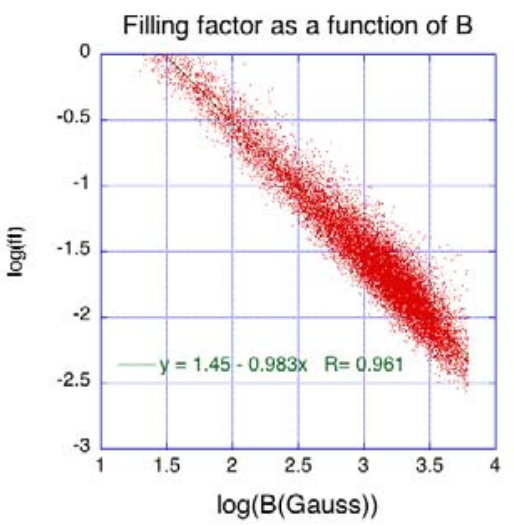

Fig. 6. Behavior of the magnetic filling factor $\alpha$ as a function of the magnetic field strength $B$. The linear fit can also be expressed as $\alpha=$ $B_{1} / B$, with $B_{1}=28 \mathrm{G}$.

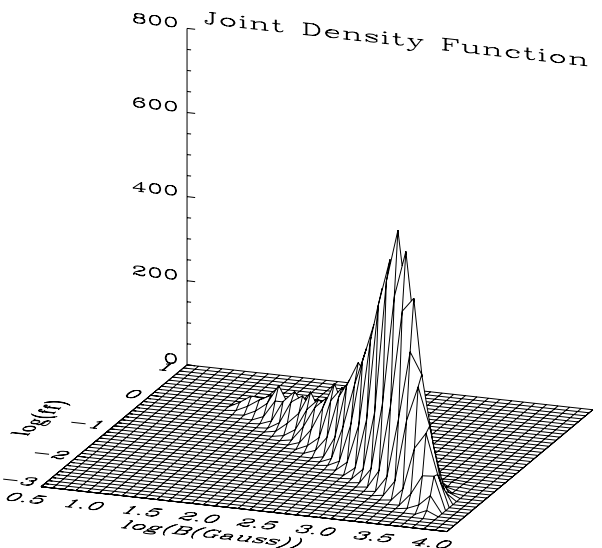

Fig. 7. 2D histogram of the magnetic filling factor and of the magnetic field strength $B$, each pair of them known in each solar pixel. This figure is a 3D representation of the number of points in Fig. 6. The joint PDF $p(\alpha, B)$ is the envelope of the $2 \mathrm{D}$ histogram.

\section{Determination of the magnetic filling factor}

The magnetic filling factor was determined following the same method as in Bommier et al. (2009). The results are given in Figs. 5-9 to be respectively compared to Figs. 10, 12-15 of Bommier et al. (2009). The behaviors are extremely similar, 


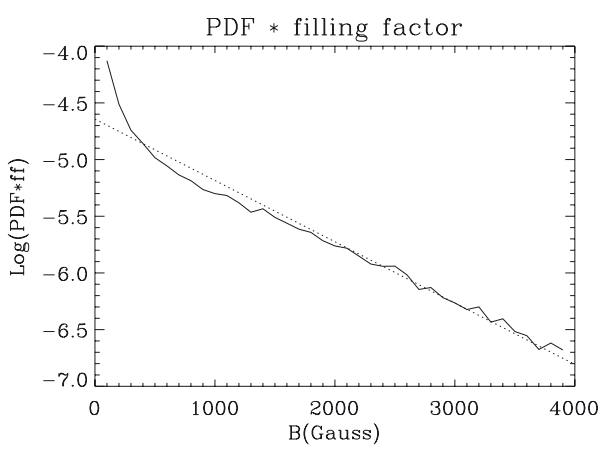

Fig. 8. Weighted histogram of the magnetic field strength. For each bin of the magnetic field strength, the count number has been multiplied by the average magnetic field filling factor for this magnetic field strength $\alpha=B_{1} / B$, with $B_{1}=28 \mathrm{G}$ following the linear fit in Fig. 6 . The envelope of this histogram is then $\bar{\alpha}_{B} p(B)$, where $\bar{\alpha}_{B}$ is the average filling factor conditioned by $B$, and $p(B)$ is the magnetic field PDF of Fig. 9. The linear fit corresponds to an exponential decrease of $\bar{\alpha}_{B} p(B)$ as $\exp \left(-B / B_{0}\right)$, with $B_{0}=803 \mathrm{G}$.

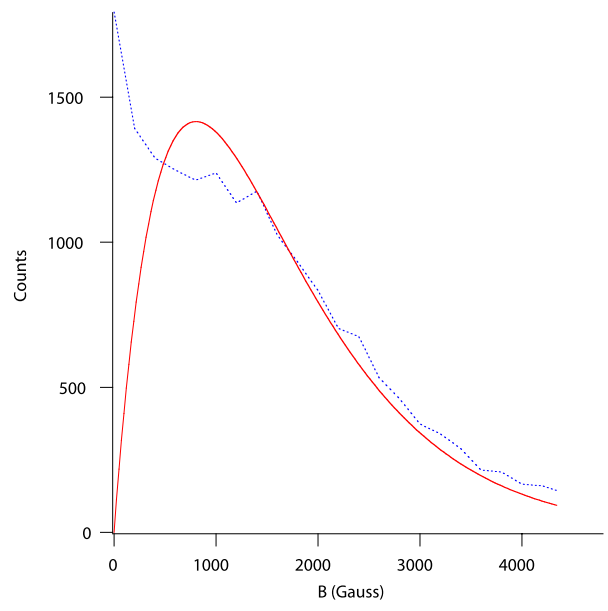

Fig. 9. Histogram of the magnetic field strength (dotted line) and fit by the magnetic field PDF $p(B)$ derived from the linear fits of Figs. 6 and 8. This magnetic field PDF is given by Eq. (18) of Bommier et al. (2009).

leading to the same relation between the magnetic filling factor $\alpha$ and the magnetic field strength $B$

$\alpha=\frac{B_{1}}{B}$,

with the somewhat different value $B_{1}=28 \mathrm{G}$ (instead of $15 \mathrm{G}$ ) and to the magnetic field strength PDF

$p(B)=\frac{B}{B_{1} B_{0}\left(1+B_{0} / B_{1}\right)} \mathrm{e}^{\frac{B_{1}-B}{B_{0}}}$,

with the slightly different value $B_{0}=803 \mathrm{G}$ (instead of $660 \mathrm{G}$ ). Again, we ascribe these value differences to the different pixel sizes of the two observations.

\section{Search for a correlation length}

Finally, we computed the correlation length along the slit for the inclination and azimuth of the magnetic field vector inferred from the inversion. The autocorrelation functions $P_{X}( \pm L)$ have been plotted in Fig. 10 for the THEMIS/THEMIS data and
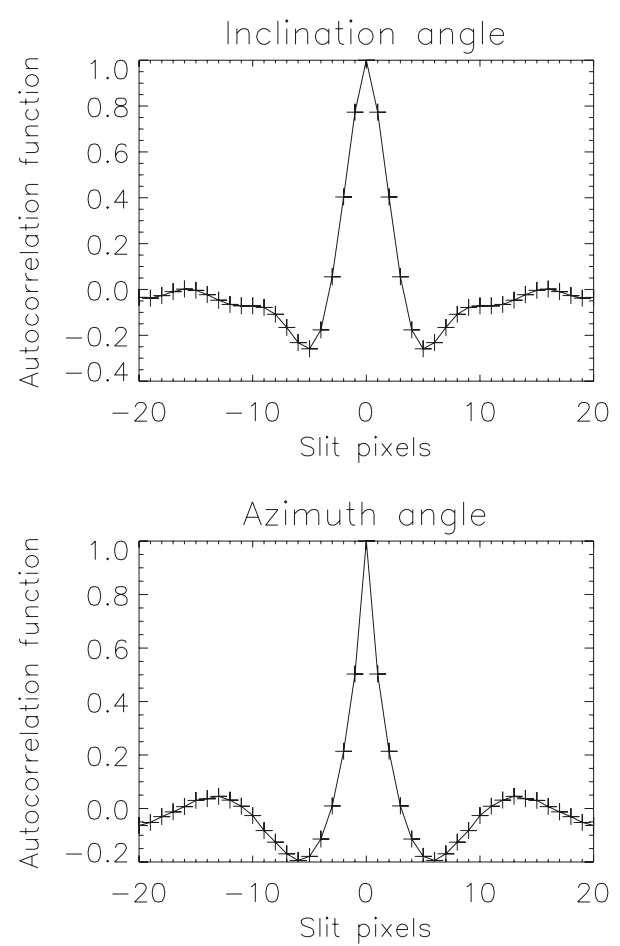

Fig. 10. Spatial autocorrelation of the field inclination and azimuth for the observations of 25 July 2007 . The abscissa is the pixel along the slit. The pixel size was 0.21 arcsec.

Fig. 11 for the ZIMPOL/THEMIS data, as a function of the distance $L$ in pixel numbers (pixels along the slit), following

$P_{X}( \pm L)=\frac{\sum_{k=0}^{N-L-1}\left(x_{k}-\bar{x}\right)\left(x_{k+L}-\bar{x}\right)}{\sum_{k=0}^{N-1}\left(x_{k}-\bar{x}\right)^{2}}$,

where $x_{k}$ is the value taken by the variable $X$ at pixel $k$, and $\bar{x}$ is the average over all the pixels. It can be seen that an average correlation length of about $0.35 \operatorname{arcsec}(250 \mathrm{~km})$ can be derived from all the figures, the THEMIS/THEMIS (Fig. 10) data where the pixel size is 0.21 arcsec as well as the ZIMPOL/THEMIS data (Fig. 11) where the pixel size is 0.53 arcsec. More precisely, the half-width at half-height is 2 pixels for the inclination, and 1 pixel for the azimuth for the THEMIS/THEMIS data in Fig. 10 where the pixel size is 0.21 arcsec. And the half-width at half-height is 1 pixel for the inclination (with respect to the broadening far wings that we estimate unphysical) and 0.5 pixel for the azimuth for the ZIMPOL/THEMIS data of Fig. 11 where the pixel size is 0.53 arcsec. We averaged all these values.

That the two series of independent data lead to close values is also an argument that this spatial correlation is true.

\section{Discussion}

\subsection{An organized magnetic field structure instead of a turbulent one}

Figure 12 displays scatter plots of the magnetic field strength as a function of the field inclination obtained in eah pixel. The result is that these two quantities are found not to be independent for both the THEMIS/THEMIS and ZIMPOL/THEMIS data. Figure 13 is the same plot but in the form of a 2D histogram. These data are the same as the ones that give the inclination 

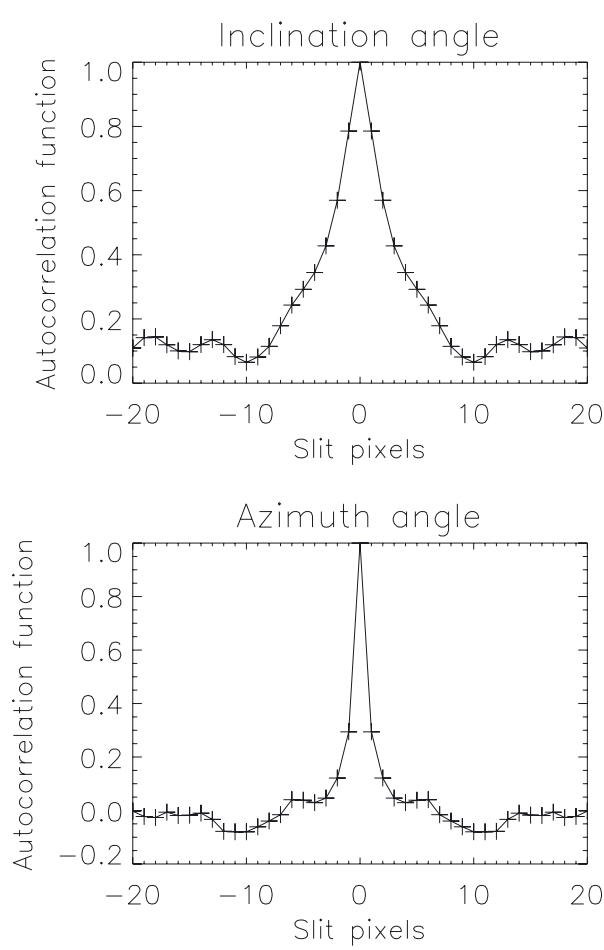

Fig. 11. Spatial autocorrelation of the field inclination and azimuth, for the observations of 5 and 6 July 2008 performed at disk center with the ZIMPOL polarimeter. The abscissa is the pixel along the slit. The pixel size was 0.53 arcsec.

histogram of Figs. 1 of the present paper and 4 of Bommier et al. (2009). When we plotted these figures, we were expecting a shapeless cloud of points, based on the usually admitted model of the turbulent field for the internetwork field. On the contrary, the plot displayed an organized structure, where the strongest fields are found more vertical, and the weakest fields are found to be more horizontal. It has to be recalled that we also find that the strongest fields correspond to the smallest magnetic filling factors (see Eq. (2)). Thus, this leads to the structure of scattered narrow vertical flux tubes with a strong field which open and weaken with height, but inside the photosphere, so that the result of the opening, which is the weak field, is also present as a horizontal field in the photosphere. Such a 2D horizontal weak field is a possible alternative interpretation of the Hanle effect measurements in limb polarization that were previously interpreted in terms of a 3D turbulent field.

It has to be recalled that the results of our measurements are strongly based on the model used for interpreting each pixel's data. Each pixel is assumed to be filled by a 2-component atmosphere, one without a magnetic field, the other with the magnetic field $B$ and filling the fraction $\alpha$ of the whole space. The nonmagnetic atmosphere fills the $1-\alpha$ fraction. For instance, the probability of observing a given field strength $B$ (lying between $B$ and $B+\mathrm{d} B)$ is the relative number of times that this value is obtained in a series of pixels, each pixel being interpreted in the aforementioned modeling. The PDF is the probability of the result of the measurement (that contains a modeling for the interpretation), and is not the spatial function $B(x, y, z)$ that really describes the magnetic field.

A priori, our modeling is unable to discriminate between the $\alpha$ fraction achieved by a single fluxtube, and the same fraction achieved by several fluxtubes occupying each the fraction $\alpha_{1}, \alpha_{2}, \ldots, \alpha_{N}$ such that $\alpha=\alpha_{1}+\alpha_{2}+\ldots+\alpha_{N}$. However, we
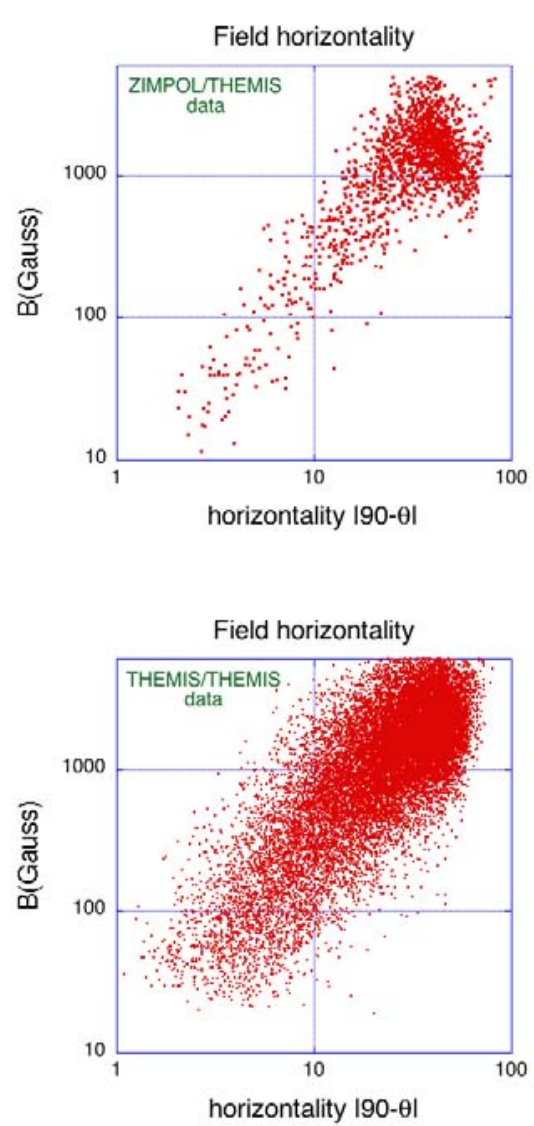

Fig. 12. An organized structure: magnetic field strength as a function of the angle between the field and the horizontal plane. Top: data obtained in July 2008 with the ZIMPOL polarimeter mounted on the THEMIS telescope and described in Bommier et al. (2009). Bottom: data obtained in July 2007 with the THEMIS polarimeter and described in the present paper.

detected a non-zero spatial correlation between neighboring pixels, which is described in Sect. 6. That a spatial correlation between pixels is observed speaks in favor of the model of the single (or few) fluxtubes filling the $\alpha$ fraction of the pixel. This is coherent with the fact that the Zeeman effect is a linear effect, so that the observed effect is the average effect and is also the effect of the average field. Thus, if the spatial structure of the field was much smaller than the pixel size, no effect would be observed. That an average Zeeman effect is observed supports also the model of the single (or a small number of) fluxtubes in each pixel. This seems to somewhat contradict the conclusion of Martínez González et al. (2010b), who claim a cascade of spatial scales within the resolution element for the magnetic field. Their conclusion is based on a comparison of circular polarization signal amplitudes at different spatial resolutions. They claim a common behavior of the histograms demonstrating this cascade, but as for us we see differences between the different spatial scales in their Figs. 2 and 3.

The present result of a strong vertical field that weakens in becoming horizontal is strongly analogous with the recent discovery of small magnetic field loops in the quiet-Sun atmosphere (Martínez González et al. 2010a; Danilovic et al. 2010). 


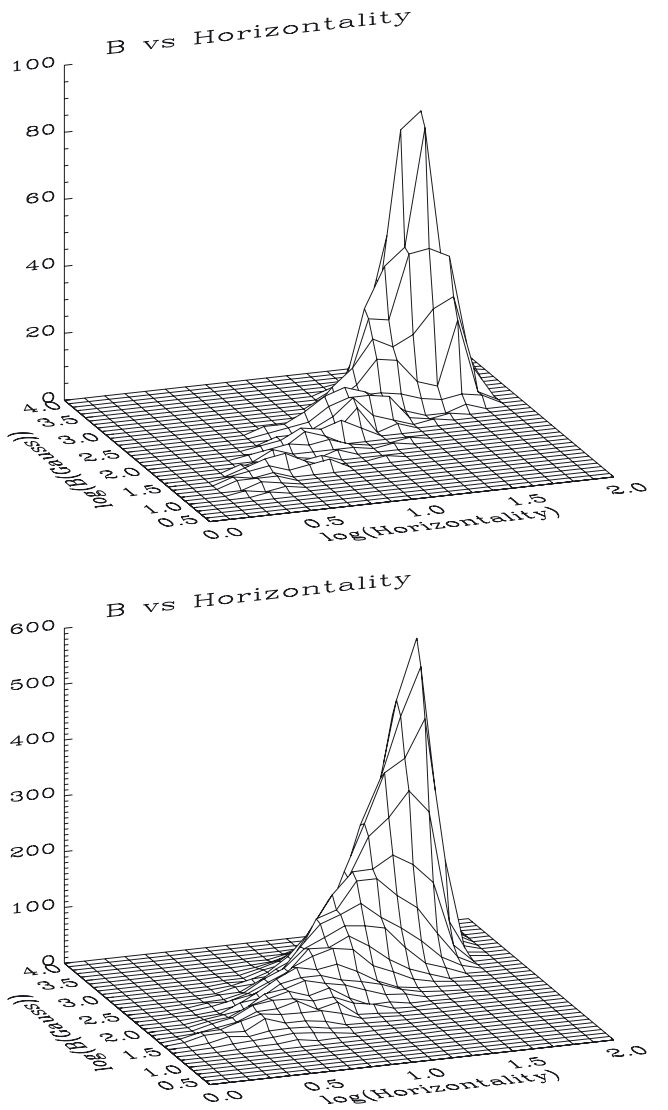

Fig. 13. 2D histogram of the magnetic field strength as a function of the angle between the field and the horizontal plane (same as Fig. 12, but in 2D histograms). Top: ZIMPOL/THEMIS data. Bottom: THEMIS/THEMIS data.

\subsection{Return on the discussion about the magnetic filling factor modeling in the data inversion}

Finally, we have to go deeper into the comparison between the different methods used in the literature to model the magnetic filling factor in the data inversion. From Hinode data interpreted with an Milne-Eddington (ME) inversion method derived from the pioneer work of Skumanich \& Lites (1987), Orozco Suárez et al. (2007) find a most probable field strength of about $100 \mathrm{G}$ associated to a magnetic filling factor of $20 \%$, whereas we find a maximum PDF for $B$ of about $800 \mathrm{G}$ filling a few percent of space (typically $2 \%$ ). We have already discussed this difference in a previous paper (Bommier et al. 2009), but more recent work has been devoted either to confirming the hG field by using a different inversion method (Asensio Ramos 2009) or to performing tests of the inversion method that also confirms the hG result (del Toro Iniesta et al. 2010). In light of these recent works, we have to go back to this discussion.

First, it has to be pointed out that all the works agree on the $\alpha B$ product. The field strength of about $100 \mathrm{G}$ associated to the magnetic filling factor of $20 \%$ (Orozco Suárez et al. 2007) corresponds to about $20 \mathrm{G}$ for $\alpha B$, whereas we get $15 \mathrm{G}$ in Bommier et al. (2009) and $28 \mathrm{G}$ in the present work. All these values agree. The difference lies in the modeling of the non-magnetic part of the atmosphere for the inversion. In our work, we assume that all physical parameters of the magnetic and non-magnetic parts of the atmopshere are equal, except for the presence/absence of the magnetic field, and we derive the set of physical parameters plus magnetic field in one inversion step, as described in Bommier et al. (2007). In the other works, it is not assumed that the magnetic and nonmagnetic parts of the atmosphere have the same physical parameters, and the problem is to determine the "non-magnetic intensity", i.e. the intensity of the radiation emitted by the nonmagnetic part of the atmopshere. This intensity has to be determined in a preliminary step, and once this intensity is fixed, the Marquardt algorithm is performed for the inversion. In the pioneering work of Skumanich \& Lites (1987) and related works where the observations were centered on active regions, the nonmagnetic intensity was taken as the intensity profile averaged on the quietest part of the map or by averaging the observed intensity profile on the whole map. In their quiet-Sun observations, Orozco Suárez et al. (2007) derived the nonmagnetic intensity profile from a preliminary average on the eight pixels that surround each pixel. However, it has to be mentioned that the intensity profile does not behave linearly with the magnetic field, because the magnetic field can only broaden the profile and never narrow it, so that the average profile is not the profile corresponding to the average field. This last profile, if known, should be taken as the one of the nonmagnetic atmosphere, but it cannot be else than different from the profile obtained by averaging the observations, which is usually taken in place. Both Orozco Suárez et al. (2007) and Asensio Ramos (2009) apply this method (averaging on the eight surrounding pixels) to determine the nonmagnetic profile, so that it is normal that their final results are in close agreement (hG fields). Coming back to our approach, we showed by a test performed in Bommier et al. (2007) that $\alpha$ and $B$ cannot be separately determined from ME inversion of a single line, but that their product $\alpha B$ is properly recovered, as also shown by the comparison above. More precisely, this only concerns the weaker field values (smaller than $1 \mathrm{kG}$ ), and occurs because there is noise in the data. When the test is performed on unnoisy theoretical data, the separate retrieval of $\alpha$ and $B$ is successful. Tests of the alternative approach (the one where the nonmagnetic profile is previously determined by averaging) have recently been performed by del Toro Iniesta et al. (2010). They conclude that the separate retrieval of $\alpha$ and $B$ is possible, but a close inspection of their Fig. 6 shows that this is only true for strong field, as in our work. Finally, all the analyses find $\alpha B$ in the $15-28 \mathrm{G}$ range, which is the only conclusion possible from the inversion. In the present work, we added an independent evaluation of the filling factor that permits a final evaluation of the magnetic field strength that we got in the $\mathrm{kG}$ range and not in the hG range, but it has to be kept in mind that all the works agree and that the difference lies in the modeling of the filling factor in the inversion.

Interesting is comparison with the recent results of Viticchié et al. (2011). They analyze HINODE data of a quiet Sun field of view. They apply the MISMA model that takes the inhomogeneous structure of the magnetic field into account inside the pixel by considering the mixed contribution of three components: one free of magnetic field and two with a magnetic field having the same inclination but different strengths. For signal level considerations, only $2.3 \%$ of their pixels were fully Stokes-analyzed, so we discard them in the present discussion. In all the other analyzed pixels (29\% of the whole pixels), the field was assumed to be vertical. Their results are very close to ours, as for the vertical field. They get a filling factor $\alpha=2.3 \%$ for it in the internetwork (see their Table 1), corresponding to a most probable field strength of $900 \mathrm{G}$ (see their Fig. 8) at the formation height of the $\mathrm{Fe}_{\mathrm{I}}$ lines that we both analyzed. This $900 \mathrm{G}$ value is in excellent agreement with the maximum value of our magnetic field PDF (see our Fig. 9). From the law of our Eq. (2) this $900 \mathrm{G}$ value has a filling factor of $3.1 \%$, which is also not far from the maximum value $\alpha=2 \%$ of our filling factor histogram of Fig. 5, taking the 
inaccuracy of our filling factor determination into account. As is visible in Fig. 12, this field strength of $900 \mathrm{G}$ also corresponds to vertical fields in our analysis, in agreement with the MISMA model. Accordingly, their average flux density determination of $19 \mathrm{G}$ is in very good agreement between our values of $13.5 \mathrm{G}$ (ZIMPOL analyzer) and $27 \mathrm{G}$ (THEMIS analyzer).

The field strength histogram of Fig. 8 of Viticchié et al. (2011) shows, however, a sharp decrease for fields stronger than $1000 \mathrm{G}$. In contrast our histogram of Fig. 9 displays fields stronger than $2000 \mathrm{G}$, which is too strong for an equilibrium between magnetic flux tubes and field-free environment. This distribution tail comes from our filling factor determination only being approximative. As already stated, the inversion is unable to determine separately the magnetic filling factor and magnetic field strength, only their product is determined at the inversion step. We then determined the filling factor (from which we derive the field strength from the product) by an approximate method that remains inaccurate. Refinement of our filling factor determination from the Stokes profiles is our current project, and the two component unresolved pixel model with one nonmagnetic and one magnetic component, certainly also needs to be refined as suggested by the present three component MISMA approach.

\section{Conclusion}

The major conclusion of this paper is that the field inclination depends on the field strength, so that the field structure appears as scattered, narrow vertical flux tubes with strong field (typical $800 \mathrm{G}$ ), which open and weaken with height, but do it inside the photosphere, so that the weak field is present as a horizontal field in the photosphere. The structure of the weakest part of the field is then 2D (horizontal) instead of 3D (turbulent). This does not contradict the observations of Hanle depolarization, because the Hanle effect in limb scattering is insensitive to vertical fields (aligned with the anisotropy of the incident radiation), so that a 2D horizontal weak field leads to a similar Hanle depolarization, which is also a possible interpretation of these observations. The study of the spatial correlation between the field vector inclination and azimuth along the different pixels speaks in favor of the model of a single (or few number of) fluxtube filling the $\alpha$ fraction of the pixel, whose most probable value is $\alpha=2 \%$ (maximum of Fig. 5). The correlation length is found to be approximately $250 \mathrm{~km}$.
Acknowledgements. The author is very grateful to M. J. Martínez González for having proposed these observations when she was a postdoc at LERMA and to the THEMIS team (B. Gelly, A. López Ariste and C. Le Men) for their help and efficiency. She is also very grateful to an anonymous referee for a careful reading of the manuscript and helpful suggestions. Thanks also to J. Adams for English language improvements.

\section{References}

Asensio Ramos, A. 2009, ApJ, 701, 1032

Bommier, V., \& Molodij, G. 2002a, A\&A, 381, 241

Bommier, V., \& Rayrole, J. 2002b, A\&A, 381, 227

Bommier, V., Derouich, M., Landi Degl'Innocenti, E., Molodij, G., \& Sahal-Bréchot, S. 2005, A\&A, 432, 295

Bommier, V., Landi Degl'Innocenti, E., Landolfi, M., \& Molodij, G. 2007, A\&A, 464, 323

Bommier, V., Martínez González, M., Bianda, M., et al. 2009, A\&A, 506, 1415

Danilovic, S., Beeck, B., Pietarila, A., et al. 2010, ApJ, 723, L149

del Toro Iniesta, J. C., Orozco Suárez, D., \& Bellot Rubio, L. R. 2010, ApJ, 711, 312

Domínguez Cerdeña, I., Sánchez Almeida, J., \& Kneer, F. 2003, A\&A, 407, 741 Faurobert-Scholl, M. 1993, A\&A, 268, 765

Faurobert-Scholl, M., Feautrier, N., Machefert, F., Petrovay, K., \& Spielfiedel, A. 1995, A\&A, 298, 289

Khomenko, E., \& Collados, M. 2007, ApJ, 659, 1726

Khomenko, E. V., Collados, M., Solanki, S. K., Lagg, A., \& Trujillo Bueno, J. 2003, A\&A, 408, 1115

Lites, B. W., \& Socas-Navarro, H. 2004, ApJ, 613, 600

López Ariste, A., Martínez González, M. J., \& Ramírez Vélez, J. C. 2007, A\&A, 464, 351

Martínez González, M. J., Collados, M., \& Ruiz Cobo, B. 2006, A\&A, 456, 1159 Martínez González, M. J., Collados, M., Ruiz Cobo, B., \& Beck, C. 2008, A\&A, 477, 953

Martínez González, M. J., Manso Sainz, R., Asensio Ramos, A., \& Bellot Rubio, L. R. 2010a, ApJ, 714, L94

Martínez González, M. J., Manso Sainz, R., Asensio Ramos, A., López Ariste, A., \& Bianda, M. 2010b, ApJ, 711, L57

Orozco Suárez, D., Bellot Rubio, L. R., del Toro Iniesta, J. C., et al. 2007, ApJ, 670, L61

Rees, D. E., \& Semel, M. D. 1979, A\&A, 74, 1

Skumanich, A., \& Lites, B. W. 1987, ApJ, 322, 473

Socas-Navarro, H., \& Sánchez Almeida, J. 2002, ApJ, 565, 1323

Socas-Navarro, H., Martínez Pillet, V., \& Lites, B. W. 2004, ApJ, 611, 1139

Stenflo, J. O. 1982, Sol. Phys., 80, 209

Stenflo, J. O., \& Holzreuter, R. 2003, in Current Theoretical Models and Future High Resolution Solar Observations: Preparing for ATST, ed. A. A. Pevtsov, \& H. Uitenbroek, ASP Conf. Ser., 286, 169

Trujillo Bueno, J., \& Manso Sainz, R. 1999, ApJ, 516, 436

Trujillo Bueno, J., Shchukina, N., \& Asensio Ramos, A. 2004, Nature, 430, 326

Viticchié, B., Sánchez Almeida, J., Del Moro, D., \& Berrilli, F. 2011, A\&A, 526, A60 